\title{
DESENVOLVIMENTO DE UM SENSOR VIRTUAL PARA ESTIMAR A TAXA DE RECUPERAÇÃO DE MINÉRIO USANDO REDE NEURO-FUZZY*
}

\author{
Jedson Alessandro Damasceno ${ }^{1}$ \\ Wagner Francisco Marques ${ }^{2}$ \\ Rogério Cosendey Labanca ${ }^{3}$
}

\section{Resumo}

Atualmente, a maioria dos pátios utilizam recuperadoras com roda de caçambas para remover o minério das pilhas de estocagem. Tipicamente, estas máquinas são operadas manualmente, com todos os movimentos controlados por operadores, o que faz com que o processo seja totalmente dependente de sua experiência, tornando difícil manter o processo em operação ótima. Para alcançar uma melhor performance, foi implementado um sistema automático que controla todos os movimentos de recuperação. Um importante desafio encontrado durante a construção dessa solução foi a de estimar a taxa de recuperação em tempo real, uma vez que esta informação é essencial para o algoritmo que controla o movimento de giro da máquina. Uma medição real dessa taxa está disponível, mas, a balança localizada na lança fica distante da roda de caçambas. Em outras palavras, a medição real está disponível com um grande atraso (aproximadamente 13 segundos). Este delay é inaceitável para o algoritmo de controle que necessita de uma medida em tempo real da taxa de recuperação na roda de caçambas. Diante disso, foi necessário implementar um sensor virtual. Este sensor foi implementado usando um sistema neuro-fuzzy para estimar a taxa de recuperação em tempo real utilizando outras variáveis disponíveis, tais como a pressão na roda de caçambas e medidas de penetração obtidas por radares. As estimativas obtidas ficaram muito próximas dos valores reais, e este sensor pôde então ser utilizado pelo algoritmo de controle.

Palavras-chave: Sensor virtual; Neuro-fuzzy; Estimador; Recuperação de minério.

\section{DEVELOPMENT OF A SOFT SENSOR TO ESTIMATE ORE RECOVERY RATE USING NEURO-FUZZY NETWORK}

\begin{abstract}
Currently, the majority of ore stockyards uses bucket wheel reclaimers to remove the ore from the stockpiles. Typically, these machines are operated manually, with all machine movements controlled by operators, which makes the process totally dependent upon their experience, making it difficult to keep the process within the optimal operating points. To achieve a better performance, it was implemented an automatic system that controls all recovery movements. An important challenge found during the construction of this solution was to estimate the recovery rate in real time, since this information is essential for the algorithm that controls the machine's slew movement. A real measurement of this rate is available, but the belt scale in the boom is far from the bucket wheel. In other words, the real measurement is available with a big delay (about 13 seconds). This delay is unacceptable to the control algorithm that needs a real time measurement of the recovery rate in the bucket wheel. In this case, it was necessary to implement a soft sensor. The soft sensor was implemented using a neuro-fuzzy system to estimate the real time recovery rate using other available measurements, like the pressure in the buckets and radar measurements. The results obtained from this soft sensor were very near from the real sensor and they could be used by the control algorithm.
\end{abstract}

Keywords: Soft sensor; Neuro-fuzzy; Estimation model; Ore recovery.

Engenheiro Eletricista, M.Sc., Gerente Sênior, Accenture, Belo Horizonte, MG, Brasil.

Técnico em Eletrônica, Consultor Sênior, Accenture, Belo Horizonte, MG, Brasil.

Engenheiro de Controle e Automação, PMP, Consultor Sênior, Accenture, Belo Horizonte, MG, Brasil. 


\section{INTRODUÇÃO}

O objetivo da recuperação automática, realizada por recuperadoras em pátios de minério de ferro, é manter a taxa de recuperação a mais eficiente possível durante a recuperação e reduzir sua variabilidade, principalmente durante as inversões de giro nas bordas da pilha (Figura 1) (Damasceno [1,2]).

Dentro deste algoritmo de controle existe uma função de alta complexidade que é responsável por calcular o fluxo real produzido, antes da medição real, a qual ocorre vários segundos depois do contato entre a roda de caçambas da recuperadora e a pilha.
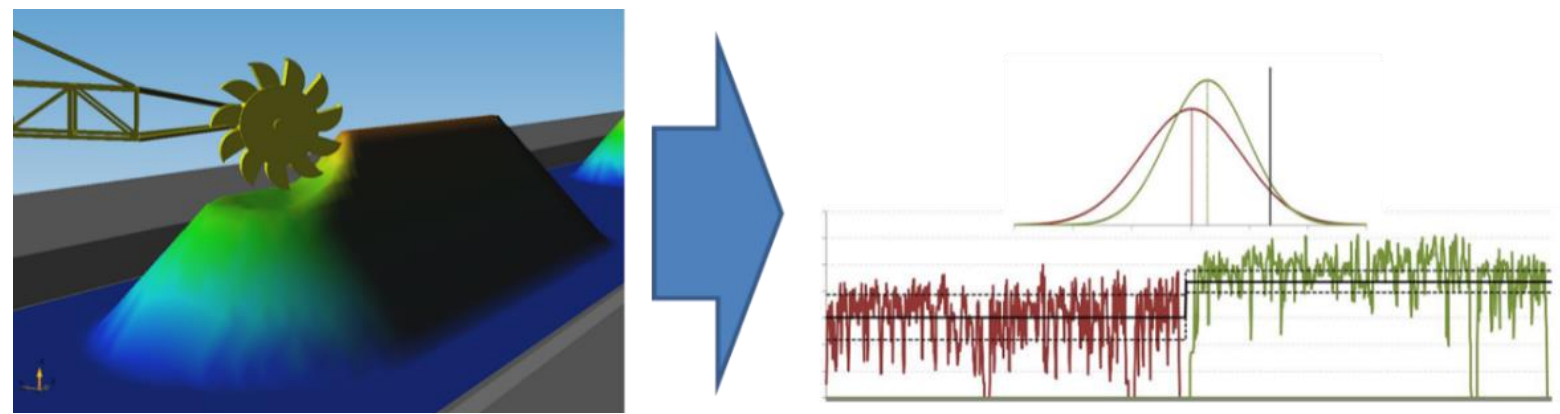

Figura 1. Redução na variabilidade do processo obtida através da recuperação automática.

A balança que faz a medição da taxa de recuperação da máquina se encontra instalada na correia transportadora da lança. Isto por questões de manutenção (local mais protegido de projeção de minério e interferência de outros equipamentos) e qualidade da leitura (menor perturbação da movimentação do material, causando maior estabilidade na leitura). Devido a esta localização da balança, a informação de fluxo não é utilizada no controle imediato da velocidade de giro da máquina, por ter um tempo de resposta excessivamente longo, não controlando o processo. Desta forma, utiliza-se a pressão da roda de caçambas (ou a corrente deste motor) para o controle do processo, pois, existe uma alta correlação entre o fluxo de minério e a pressão exercida na roda de caçambas durante a operação. Este controle tipicamente utiliza um controlador PID (Proporcional Integral Derivativo), sendo que a variável de processo utilizada é a pressão da roda de caçambas (ou, dependendo do tipo de acionamento, a corrente deste motor).

Para calcular a correlação entre o fluxo da balança e a pressão na roda de caçambas, eram utilizados métodos de regressão linear, que necessitavam de uma análise dos dados offline (utilizando dados históricos). Esta análise consiste na sincronização manual da pressão da roda de caçambas e o fluxo da balança (este defasamento é causado principalmente pelo tempo de transporte do material na correia transportadora entre a roda de caçambas e o local de instalação da balança), aplicação de métodos matemáticos de correlação, definição do algoritmo e sua codificação no CLP (Controlador Lógico Programável). Durante a sua execução, existe ainda uma correção de ganho do modelo, baseado no erro médio calculado, em relação ao fluxo da balança, fazendo a correção dinâmica no resultado calculado pelo algoritmo do CLP. Este ajuste tem a função de melhorar a correlação do algoritmo quando o modelo utilizado apresentar desvios em relação ao modelo préconfigurado no CLP. 


\section{DESAFIO}

A forma convencional de cálculo de correlação que era utilizada possuía alguns fatores limitadores que não conferiam sustentabilidade na qualidade da correlação.

Um ponto importante é o fato de existirem outros dados online da recuperadora que não estavam sendo considerados na correlação com o fluxo da balança, como por exemplo os dados provenientes dos radares, posição e velocidade da recuperadora. Como a análise dos dados é feita de forma offline, mudanças no comportamento da pressão em relação ao fluxo, causados por tipo de material utilizado, compactação do material, condições do tempo, etc, não eram percebidos pelo sistema, causando erros de controle e necessidade de intervenção manual para reavaliação da correlação.

Pelo fato do processo conter esta correlação variável, optou-se por utilizar uma rede neuro-fuzzy, que fosse a mais autônoma possível, sendo capaz de sincronizar todos os dados, de forma a facilitar a análise e processamento destes dados e fazer com que esta análise fosse constantemente atualizada (devido ao fato da correlação não ser fixa, isto é, de acordo com as condições do material e do processo teremos correlações diferentes).

Além destes pontos apresentados, um outro desafio era projetar uma rede neurofuzzy de baixo consumo de processamento e memória, que pudesse ser executada em um CLP, o qual possui estes recursos bem limitados em relação a servidores ou outros computadores. Esta necessidade se deve ao fato do controle da máquina ficar no CLP, pois normalmente as máquinas de pátio possuem problemas de comunicação com os demais sistemas do processo, causados pela sua própria movimentação, sendo que estes atrasos ou falhas de comunicação causariam a parada do processo, caso fosse executado remotamente.

\section{SOLUÇÃO PROPOSTA}

Para a implantação deste sistema, foram desenvolvidos dois módulos no CLP e dois módulos no servidor de treinamento da rede (computador que irá realizar o armazenamento de dados e treinamento da rede), conforme destacado na Figura 2. 


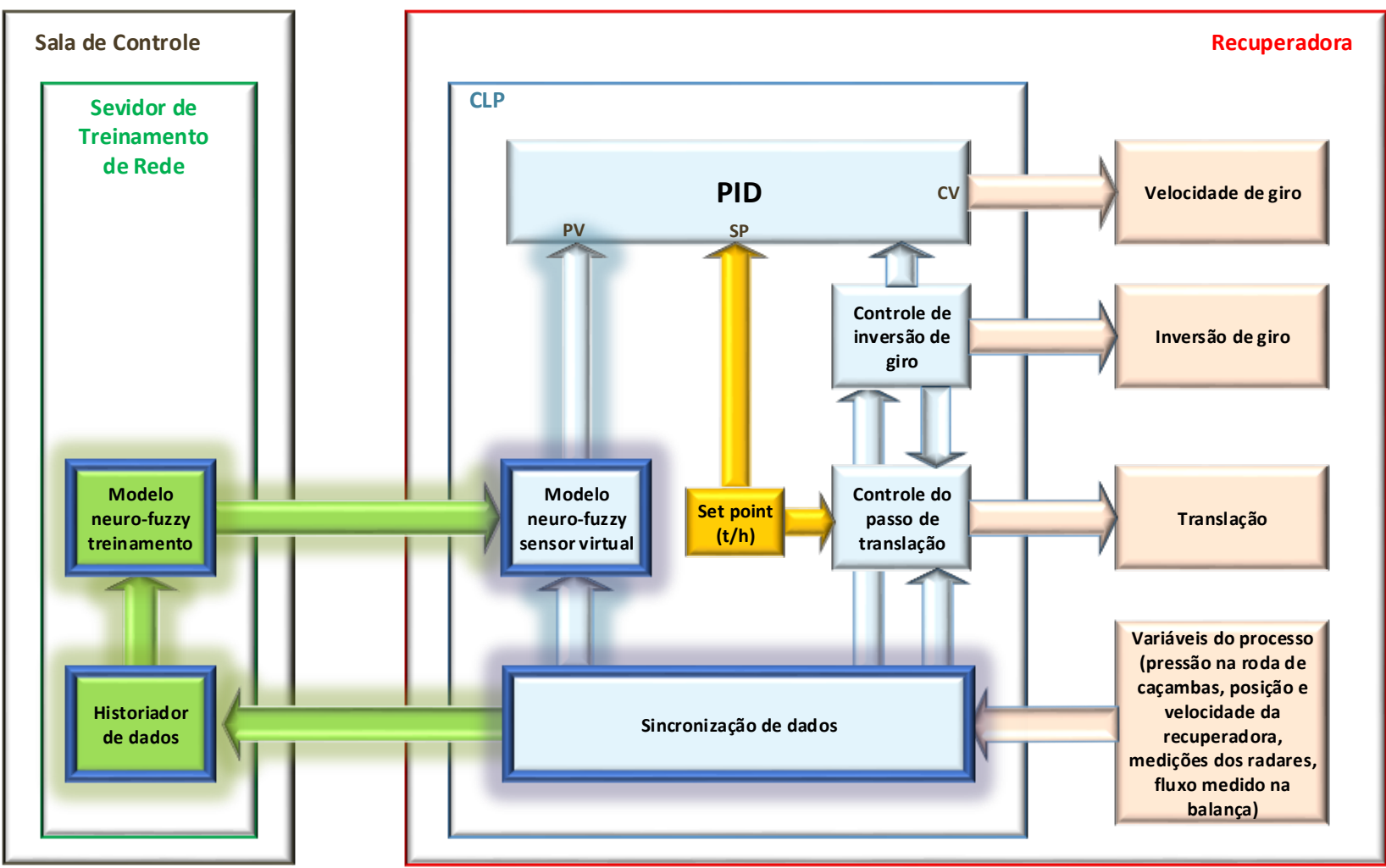

Figura 2. Associação entre controle clássico (usando PID) e inteligência artificial (rede neuro-fuzzy) para criar um controle avançado de processo.

No CLP foi implantado o módulo de sincronização de dados, o qual irá sincronizar todas as variáveis utilizadas pela rede neuro-fuzzy com o fluxo da balança. Este sincronismo irá considerar o momento da geração da leitura e sua relação com 0 fluxo da balança, considerando o tempo de transporte do material na correia transportadora, a velocidade de giro, a velocidade da roda de caçambas, etc. Este módulo irá enviar os dados sincronizados para o servidor de treinamento da rede e também para o módulo runtime, em execução no CLP.

Além do módulo de sincronização de dados, foi desenvolvido no CLP também o módulo neuro-fuzzy runtime, que utiliza os dados do módulo de sincronização, junto com os parâmetros de treinamento recebidos do servidor, possibilitando o cálculo de saída da rede neuro-fuzzy online, gerando a variável de processo (sensor virtual), para o controle no módulo PID.

No servidor de treinamento da rede foram desenvolvidos os seguintes módulos:

- O módulo de aquisição e sincronia de dados, que analisa a massa de dados de tempos em tempos para avaliação da necessidade de um novo treinamento da rede;

- E o módulo de treinamento do modelo neuro-fuzzy, responsável por treinar a rede e gerar novos parâmetros de treinamento, a serem enviados para o módulo runtime do CLP.

Com este modelo, podemos atuar no fluxo estimado, que é a inferência do fluxo instantâneo baseada em informações atuais de pressão, e também no fluxo previsto, que é baseada em informações que o radar está disponibilizando para uma condição futura.

A Figura 3 apresenta a arquitetura física da solução. 
Servidor de Treinamento da
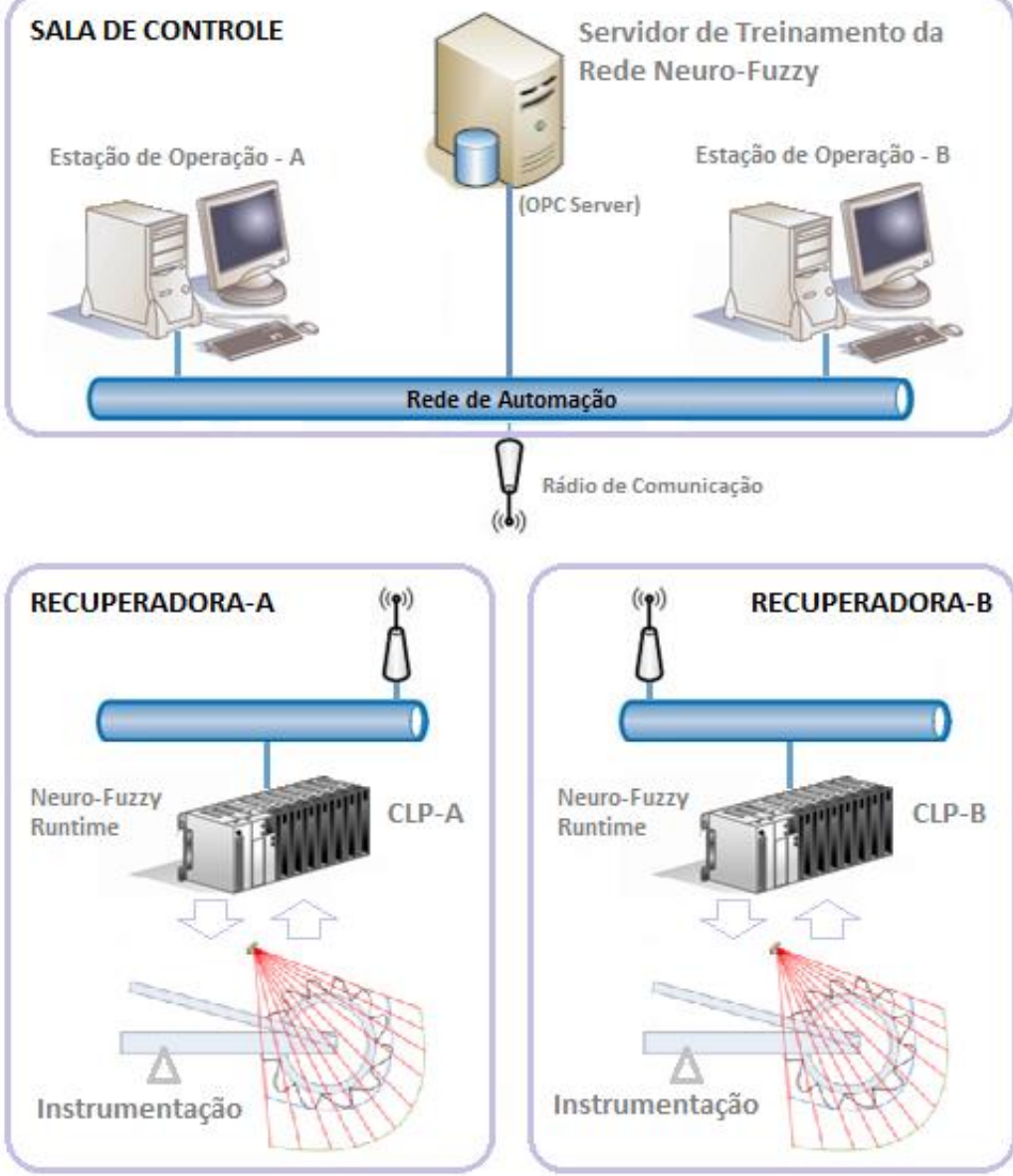

Figura 3. Arquitetura física da solução.

\section{MODELAMENTO NEURO-FUZZY}

\subsection{Estrutura da Rede Neuro-Fuzzy}

Como apresentado na Figura 2, a rede é executada em tempo real no CLP, que possui limitações de processamento e armazenamento de dados quando comparado a servidores, ou até mesmo computadores comuns. Diante disso, foi escolhida uma rede neuro-fuzzy de baixo custo computacional.

O modelo Neo-Fuzzy Neuron (NFN) foi escolhido por possuir essa característica de baixo custo, advindo do fato das funções de pertinência serem simples (triangulares e complementares). Além disso, essa rede possui apenas uma camada.

Conforme explicado por Silva [3], esse modelo pode ser visto como um conjunto de modelos do tipo Takagi-Sugeno de ordem zero, um para cada variável de entrada, cada um contendo $\boldsymbol{m}_{\boldsymbol{i}}$ regras. A saída $\boldsymbol{y}$ é obtida através da soma das saídas dos modelos individuais $\boldsymbol{y}_{\boldsymbol{i}}$, isto é

$$
y=\sum_{i=1}^{n} y_{i}=\sum_{i=1}^{n} \sum_{j=1}^{m_{i}} \mu_{i j}\left(x_{i}\right) w_{i j}
$$

onde $\boldsymbol{n}$ é o número de variáveis de entrada e $\boldsymbol{m}_{\boldsymbol{i}}$ é o número de funções de pertinência da $\boldsymbol{i}$-ésima variável de entrada, $\boldsymbol{x}_{\boldsymbol{i}}$. As funções de pertinência são triangulares e complementares e, portanto, no máximo duas das $\boldsymbol{m}_{\boldsymbol{i}}$ funções são ativadas simultaneamente para uma dada entrada $\boldsymbol{x}_{\boldsymbol{i}}$. A Figura 4 ilustra a estrutura da rede. 


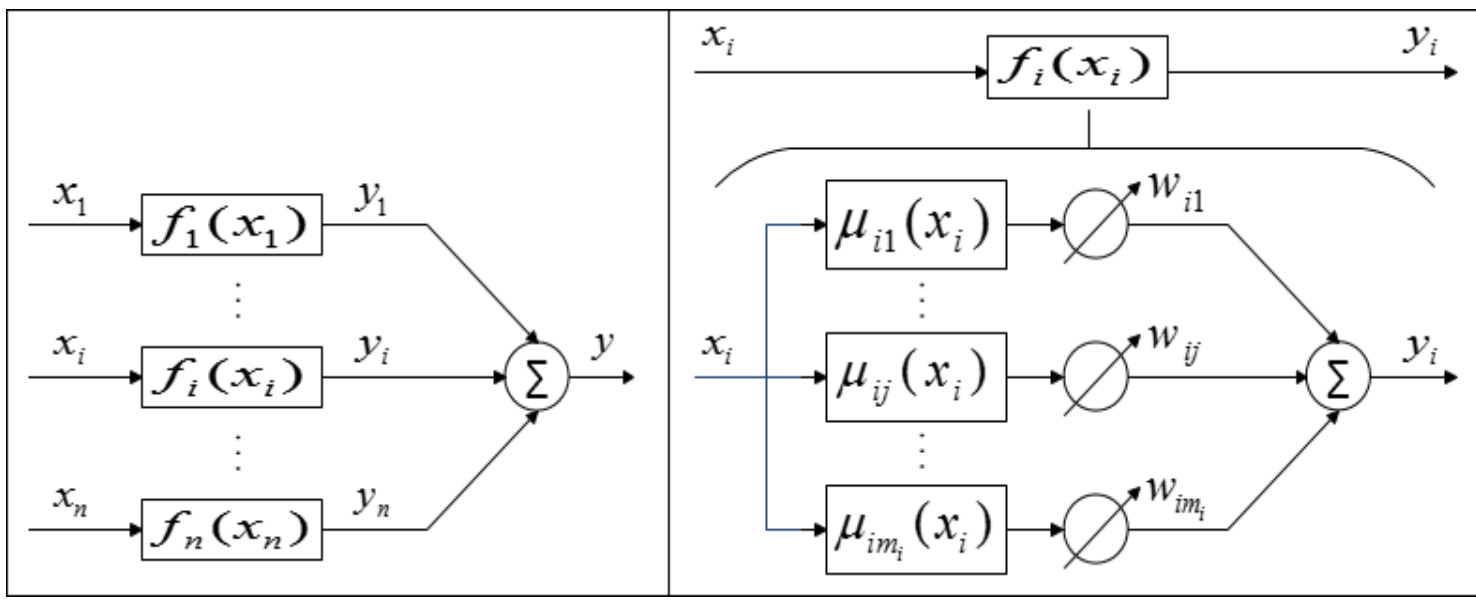

Figura 4. Estrutura da rede.

\subsection{Seleção das Variáveis de Entrada}

Uma das primeiras fases para a implementação de um modelo usando rede neurofuzzy consiste em se estudar as variáveis disponíveis do processo e verificar quais devem ser utilizadas como entradas na rede. De acordo com Piang [4], esta fase é importante, pois incluir todas as variáveis disponíveis em um modelo aumentará a complexidade deste, podendo inclusive acarretar em problemas de overfitting.

As principais variáveis disponíveis do processo são:

- Pressão na roda de caçambas;

- Penetração das caçambas na pilha;

- Altura da bancada em recuperação;

- Posição da recuperadora (translação, giro e elevação);

- Velocidade de giro.

Existem algumas técnicas que ajudam a identificar as melhores variáveis para serem utilizadas como entradas na rede. Uma delas é estudar, uma a uma, a sua associação com a variável de saída. Uma forma "visual" de se fazer isso é utilizar gráficos de dispersão. A Figura 5 mostra dois gráficos ("fluxo real x pressão na roda de caçambas" e "fluxo real x translação") com dados referentes à recuperação da base de uma pilha de minério de ferro.
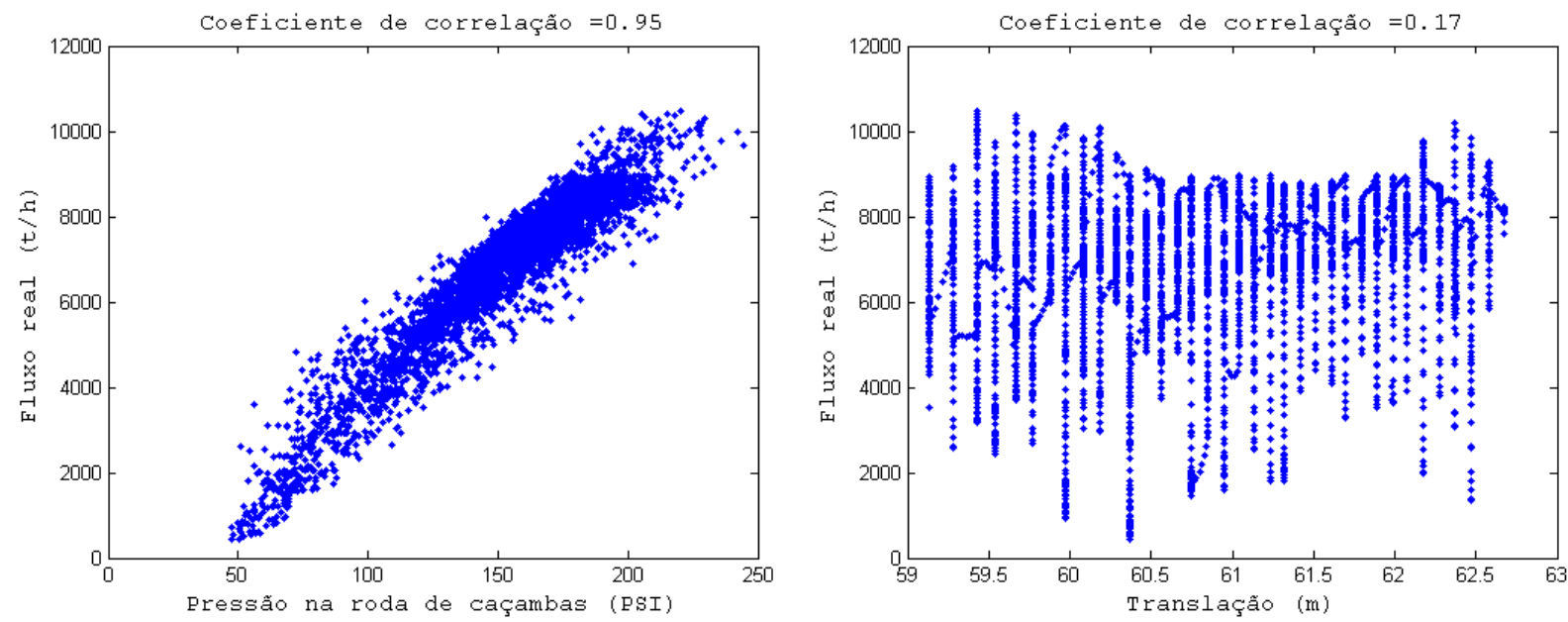

Figura 5. Gráficos de dispersão. 
Podemos observar através do gráfico "fluxo real x pressão na roda de caçambas" uma forte correlação (associação linear) entre as variáveis. O mesmo já não ocorre entre as variáveis fluxo real e translação. Podemos confirmar isto através do cálculo de coeficiente de correlação:

- $r$ (fluxo real; pressão na roda de caçambas) $=0,95$

- $r$ (fluxo real; translação

) $=0,17$

Após esse estudo utilizando gráficos de dispersão e cálculos de coeficiente de correlação, identificamos quais variáveis usar como entradas para a rede (Figura 6).

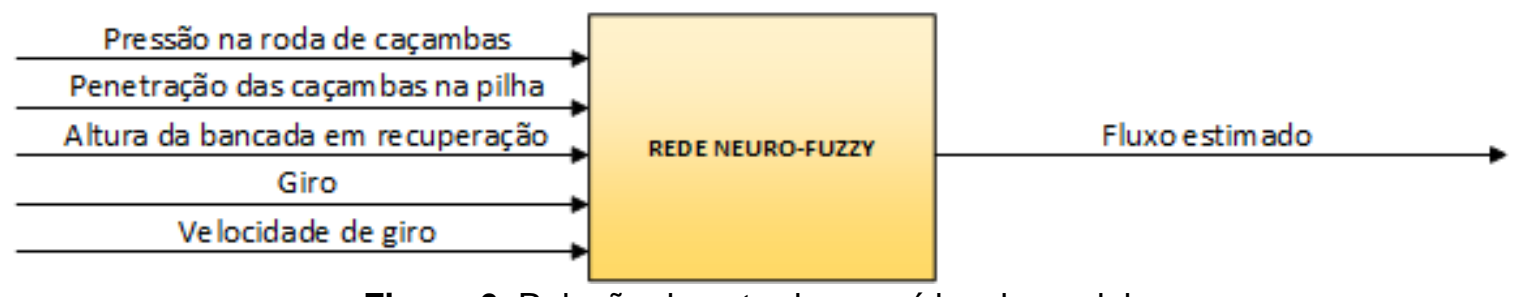

Figura 6. Relação de entradas e saídas do modelo.

Uma alternativa a este estudo de correlação entre as variáveis é aplicar o método de busca exaustiva, que consiste em realizar o treinamento e a validação da rede para cada combinação possível de entradas e utilizar algum critério de comparação (Morais Júnior [5]). Porém, esta alternativa é dispendiosa e não dá a oportunidade de se realizar um estudo mais aprofundado do processo na busca por causalidade entre cada variável e a taxa de recuperação.

\subsection{Treinamento da Rede}

O treinamento da rede (atualização dos pesos $\boldsymbol{w}_{\boldsymbol{i j}}$ ) ocorre no servidor. Isto porque o treinamento requer um alto processamento. Além disso, é no servidor que os dados utilizados ficam armazenados.

O erro do modelo é constantemente monitorado através da comparação do fluxo estimado com o fluxo real, disponível 13 segundos depois. Quando o Mean Absolute Percentage Error (MAPE) ultrapassa certo valor parametrizável, um novo treinamento utilizando dados históricos mais recentes é iniciado no servidor e o seu resultado (novos pesos) escrito no CLP via OPC (OLE for Process Control).

Ou seja, a solução implementada é auto-adaptativa, garantindo assim um resultado satisfatório do modelo mesmo com o passar do tempo. Isto é muito importante, pois a planta (seus equipamentos e instrumentos de medição) sofre deteriorização, e os sistemas auto-adaptativos absorvem essas mudanças de forma natural, ou seja, sem a intervenção de usuários ou equipe de manutenção.

\section{RESULTADOS E DISCUSSÃO}

Os resultados obtidos com o novo estimador usando rede neuro-fuzzy foram significativamente melhores em relação ao modelo anterior. A Tabela 1 apresenta um comparativo entre os dois modelos usando MAPE. De uma forma geral, pode-se dizer que o erro foi reduzido pela metade com o uso do novo estimador.

Tabela 1. Comparativo entre o modelo atual usando neuro-fuzzy e o modelo anterior

\begin{tabular}{ccc}
\hline Recuperadora & Modelo atual & Modelo anterior \\
\hline A & $<7 \%$ & $>12 \%$ \\
\hline B & $<6 \%$ & $>13 \%$ \\
\hline
\end{tabular}


A Figura 7 mostra um comparativo entre o fluxo real e o fluxo estimado pelos dois modelos ao longo de 15 minutos de recuperação pela recuperadora B.

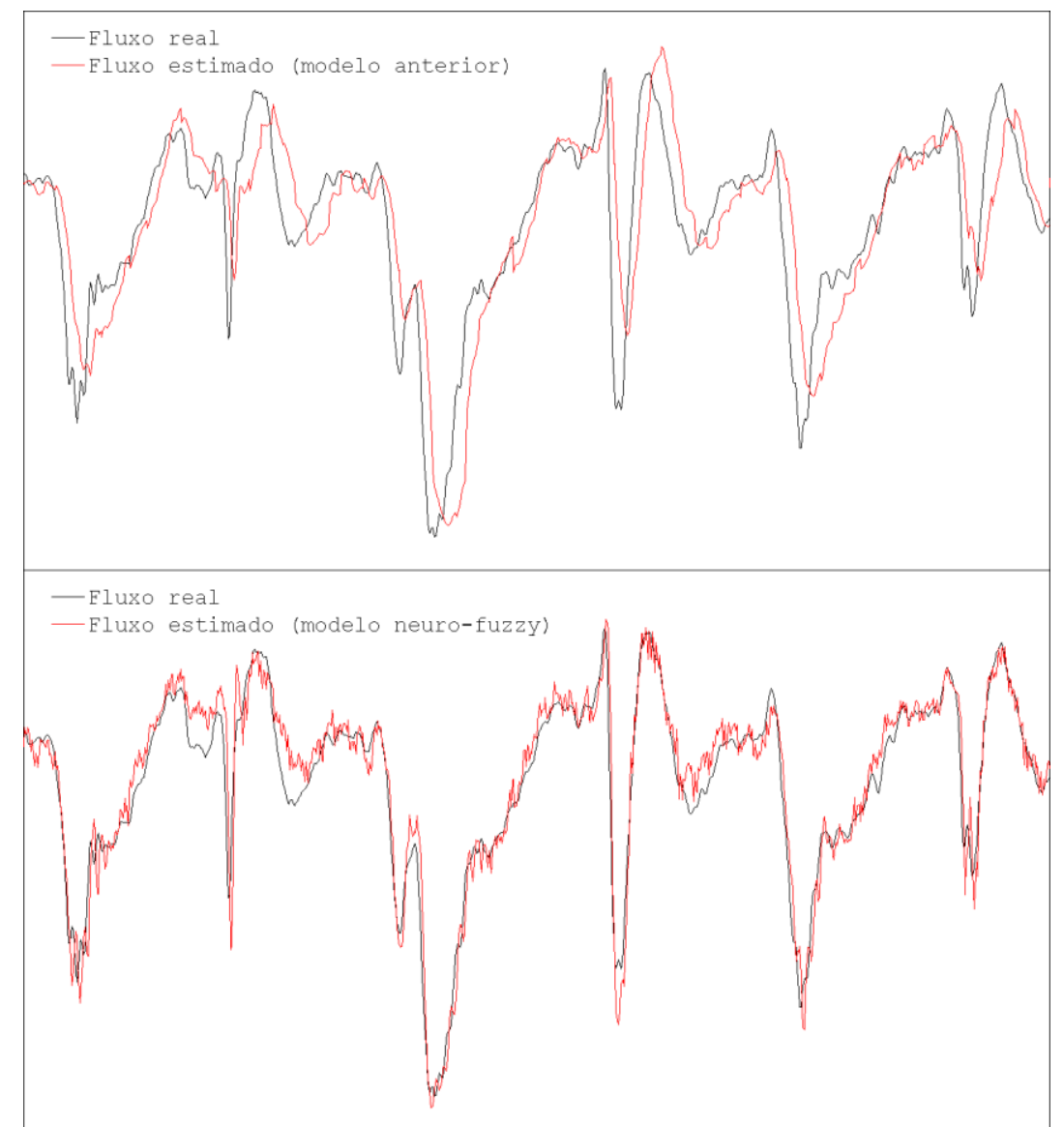

Figura 7. Comparativo entre o fluxo real e o fluxo estimado pelos dois modelos.

\subsection{Melhorias futuras}

Verificamos que parte considerável do erro do modelo refere-se ao fato do delay entre a remoção do minério pelas caçambas e a medição na balança não ser fixo em 13 segundos. Na verdade, esse atraso oscila entre 12 e 14 segundos.

Uma melhoria seria criar um algoritmo para calcular em tempo real esse delay e aplicá-lo na sincronização das variáveis de entrada e a saída (fluxo real). Desta forma, a qualidade dos dados para os treinamentos da rede seria superior, assim como a comparação "fluxo real x fluxo estimado" (cálculo do erro) seria também mais adequada.

Com essa melhoria implementada, os valores de MAPE para o modelo neuro-fuzzy apresentados na Tabela 1 seriam ainda menores.

\section{CONCLUSÃO}

Este trabalho apresentou uma abordagem baseada em uma rede neuro-fuzzy para estimar a taxa de recuperação em tempo real de duas recuperadoras de minério de ferro. Esta rede precisou ser implementada num CLP e, devido a limitações de processamento e memória, uma rede de baixo custo computacional foi escolhida.

Os resultados obtidos com esse novo estimador foram superiores ao modelo até então utilizado, que era baseado em regressão linear e utilizava apenas uma 
variável (pressão na roda de caçambas). Além disso, o sistema foi implementado de tal forma que novos treinamentos da rede pudessem ser realizados de forma automática, tão logo fosse verificado um aumento no erro do modelo.

Trabalhos futuros visam melhorias no algoritmo de sincronização de dados. Estas melhorias irão gerar amostras com qualidade superior para o treinamento da rede e um cálculo mais adequado do erro do modelo.

\section{REFERÊNCIAS}

1 Damasceno JA, Sousa LML, Labanca RC, Marques WF, Araujo FC, Lopes BE. Simulação e otimização de recuperação automática em pátio de estocagem de minério utilizando mapeamento 3D. XVII Seminário de Automação de Processos, Associação Brasileira de Metalurgia e Materiais. 2013.

2 Damasceno JA, Labanca RC, Marques WF, Augustin C, Schreiber G, Lopes BE, Costa F. From machine to MES: Full automatic stockpile management solution. Automining 2014. 2014.

3 Silva AM, Caminhas W, Lemos A, Gomide F. Rede neuro-fuzzy evolutiva para estimação em tempo real de posição de um sistema de levitação magnética. XI SBAI Simpósio Brasileiro de Automação Inteligente. 2013.

$4 \quad$ Piang LW. Inferential estimation and control of chemical processes using partial least squares based model. Thesis of Master of Engineering. Faculty of Chemical and Natural Resources Engineering, University Technology, Malaysia, 2005.

5 Morais Júnior AA. Elaboração de um analisador virtual utilizando sistema híbrido neurofuzzy para inferir a composição num processo de destilação. Dissertação de Mestrado em Engenharia Química, Universidade Federal de Alagoas, Brasil, 2011. 